\title{
Flare to care - future management of long-term conditions
}

\author{
Authors: Antony Aziz, ${ }^{A}$ Rebecca Reynolds, ${ }^{A}$ Claire Bull, ${ }^{A}$ Karen Street, ${ }^{A}$ Branita Mills, ${ }^{A}$ Patrick Kerr, ${ }^{B}$ Jeremy Hyde ${ }^{C}$ \\ and Azhar Ansari ${ }^{\mathrm{A}}$
}

\section{Aims}

To promote citizens with long-term conditions as authors of their own health data and competent self-managers supported by specialist clinical guidance that is tailored to their situation through a novel technology-driven management solution.

\section{Methods}

Through close collaborative working with patients, clinical commissioning groups and demand for an out-of-hospital experience, the inflammatory bowel disease (IBD) service at East Surrey Hospital (ESH) was radically redesigned. In 2014, a new model of care that put patients at the centre of the care pathway and opened access to all patients with IBD through telephone and email support enabled citizens to drive the change. The outcome of this led to the use of a web-based patient management portal called Patients Know Best (PKB). This synchronised the integration of patient entered symptom tracking with their own clinical information (including pathology and imaging results) and appropriate communication tools. It served as a repository for standardised patient information reducing variation among clinicians and promoting patient education. This facilitated a rapid and efficient support for patients at scale.

Patients as the assets at the centre of the change directed their own healthcare, initiated self-assessment and self-management with appropriate advice, guidance and support provided by the IBD team within 48 hours and tailored to patients' needs. The sickest patients were detected more rapidly and offered a clinical review within a week, while stable patients were offered comprehensive routine follow up non-face-to-face. Patients with IBD were invited to PKB at clinic appointments.

The service was evaluated in 2015 with data extracted from all non-face-to-face interactions that year.
Results

> In 2015, there were 4,358 non-face-to-face interactions, through which the IBD service avoided 80 hospital admissions, 134 emergency department attendances and 440 outpatient appointments.

$>1,500$ contacts related to immunosuppression therapy monitoring. There were no reported breaches of clinical safety.

> The patient journey time to access specialist care at the time of a flare reduced from 6 weeks down to 1 week.

> Fifty active users of PKB in 2015 increased to 700 in 2017 due to consumer led need and demand for a total IBD population of approximately 4,000 .

\section{Conclusion}

The redesigned IBD service at ESH is a citizen-led low-cost technology-enhanced care delivery model that has improved patient experience and clinical outcomes.

This new model of care breaks down traditional boundaries of the service user and provider, promoting collaborative working with patients with long-term conditions.

The IBD service at ESH is being delivered with lower than the national recommended number of consultants $(65 \%)$ and clinical nurse specialists (50\%), yet has provided care to more patients requiring close immunosuppression therapy monitoring than would have been possible without a non-face-to-face service, without compromising on safety. Future investment in resources can now be better focused on improving service through resilience and quality utilising co-produced mechanisms guiding the type and size of manpower to deliver patient-centred working.

\section{Conflict of interest statement}

None declared.

Authors: ${ }^{A}$ Gastroenterology, Surrey and Sussex Healthcare NHS Trust, Redhill, UK; ${ }^{\text {B}}$ East Surrey CCG, Oxted, UK; ' Patient Representative Lead, Surrey and Sussex Healthcare NHS Trust, Redhill, UK 